\title{
Mechanical Power of DC Motors with Polygarmonic Power Supply
}

\author{
Kovalov V. ${ }^{1}$, Kovalova Yu. ${ }^{2}$, Shcherbak I. ${ }^{2}$ \\ ${ }^{1}$ National Technical University "Kharkiv Politechnic Institute" \\ ${ }^{2}$ O.M. Beketov National University of Urban Economy in Kharkiv \\ Kharkiv, Ukraine
}

\begin{abstract}
The aim of the work is to develop a method for determining the mechanical power of DC motors when they are powered by semiconductor converters, since the harmonic components of the armature current do not create a torque on the shaft. This means that at the nominal electric power of the motor, it is necessary to reduce the mechanical power to ensure the nominal temperature regime of the armature winding. The aim is achieved by solving the problem of determining the dependence of the mechanical power of the motor on the armature current pulsations factor, the value of which depends on the control parameter of the thyristor or transistor converter, on the inductance of the armature circuit and the load current. As a result, it was proposed to express the dependence of the mechanical power in terms of the allowable mechanical load factor, which, in turn, depends on the armature current pulsations factor. The method is as follows: 1) on a computer model determine the armature current pulsations factor; 2) determine the allowable mechanical load factor and the allowable mechanical power. The novelty of the work lies in the fact that the expression for determining the allowable mechanical load of DC motors with polyharmonic power has been further developed, which, unlike the known ones, contains the square of the armature current pulsations factor. An example of the practical use of the proposed method for calculating the allowable mechanical power of DC motor when powered by single-phase thyristor rectifier.
\end{abstract}

Keywords: DC motor, mechanical power, load factor, factor of pulse current.

DOI: https://doi.org/10.52254/1857-0070.2022.1-53.01

UDC: 621.313.333.004.67

\section{Puterea mecanică a motoarelor de curent continuu cu alimentare poliarmonică \\ Kovaliov V.N. ${ }^{1}$, Kovaliova Iu.V. ${ }^{2}$, Scerbac I.E. ${ }^{2}$ \\ ${ }^{1}$ Universitatea tehnică Națională "Institutul politehnic din Kharkiv" \\ ${ }^{2}$ Universitatea Naţională din Kharkiv de Economie Municipală A.N. Bechetov, Harkiv, Ucraina}

Rezumat. Scopul acestei lucrări este de a elabora o metodologie pentru determinarea puterii mecanice admisibile a motoarelor de curent continuu pentru destinație industrială generală alimentate prin intermediul convertoarelor cu semiconductori, deoarece componentele armonice ale curentului rotorului nu creează cuplul motorului pe arbore. Scopul propus se realizează prin rezolvarea problemei de determinare a dependenței puterii mecanice a motorului de factorul de ondulație a curentului rotorului, a cărui valoare depinde de parametrul de comandă al convertorului cu tiristoare sau tranzistoare, de constanta de timp electromagnetică a circuitului rotorului și de curentul de sarcină. Ca rezultat al cercetărilor, se sugerează exprimarea puterii mecanice admisibile la alimentarea poliarmonică a înfăşurării rotorului prin intermediul factorului de sarcină mecanică admisibil în raport cu valoarea nominală, care, la rândul său, depinde de factorul de ondulație a curentului înfășurării rotorului. Metodica propusă constă în următoarele: 1) prin modelare la calculator a determina factorul de ondulație a curentului rotorului; 2) a determina coeficientul admisibil al sarcinii mecanice în raport cu puterea nominală; 3) a determina puterea mecanică admisibilă pe arbore. Noutatea acestei lucrări constă în faptul că a fost dezvoltată ulterior expresia pentru determinarea factorului de sarcină mecanică admisibilă la motoarele de curent continuu cu alimentare poliarmonică care, spre deosebire de cea cunoscută, conține pătratul factorului de ondulație a curentului rotorului. Este adus un exemplu de utilizare practică a metodicii oferite pentru calcularea factorului admisibil de sarcină mecanică al motorului de curent continuu la alimentarea de la redresorul monofazat pe tiristoare, ținând cont de unghiul de comandă al tiristoarelor, constanta electromagnetică a circuitului rotorului și curentul de sarcină.

Cuvinte-cheie: motor de curent continuu, putere-mecanică, coeficient de pulsații a curentului, factor de sarcină.

\section{Механическая мощность двигателей постоянного тока при полигармоническом питании Ковалев В.Н. ${ }^{1}$, Ковалева Ю.В. ${ }^{2}$, Щербак И.Е. ${ }^{2}$ \\ ${ }^{1}$ Национальный технический университет "Харьковский политехнический институт" \\ ${ }^{2}$ Харьковский национальный университет городского хозяйства имени А.Н. Бекетова Харьков, Украина}

Аннотация. Целью работы является разработка методики определения допустимой механической мощности двигателей постоянного тока общепромышленного назначения при их питании от 
полупроводниковых преобразователей, поскольку при этом гармонические составляющие тока якоря не создают крутящего момента на валу. Это означает, что при номинальной электрической мощности двигателя необходимо уменьшать механическую мощность на валу для обеспечения номинального температурного режима обмотки якоря. Поставленная цель достигается решением задачи определения зависимости механической мощности двигателя от коэффициента пульсаций тока якоря, величина которого зависит от параметра управления тиристорным или транзисторным преобразователем, от электромагнитной постоянной времени цепи якоря и тока нагрузки. В результате исследований предложено допустимую механическую мощность при полигармоническом питании обмотки якоря выражать через коэффициент допустимой механической загрузки относительно номинальной, который, в свою очередь, зависит от коэффициента пульсаций тока в обмотке якоря. Предложенная методика заключается в следующем: 1) на компьютерной модели определить коэффициент пульсаций тока якоря; 2) определить коэффициент допустимой механической загрузки относительно номинальной мощности; 3) определить допустимую механическую мощность на валу. Новизна работы заключается в том, получило дальнейшее развитие выражение для определения коэффициента допустимой механической загрузки двигателей постоянного тока при полигармоническом питании, которое, в отличие от известных, содержит квадрат коэффициента пульсаций тока якоря. Приведен пример практического использования предложенной методики для расчета допустимого коэффициента механической загрузки двигателя постоянного тока при питании от однофазного тиристорного выпрямителя с учетом угла управления тиристоров, электромагнитной постоянной цепи якоря и тока нагрузки. Полученная при этом методом планирования эксперимента аналитическая формула коэффициента пульсаций тока якоря позволяет рассчитать индуктивность сглаживающего реактора для повышения допустимой механической мощности.

Ключевые слова: двигатель постоянного тока, механическая мощность, коэффициент пульсаций тока, коэффициент загрузки.

\section{ВВЕДЕНИЕ}

В [1,2] представлен всесторонний обзор состояния вопроса в области управления электроприводами постоянного и переменного токов. Отмечается, что за последние годы достижения в области полупроводниковой силовой электроники и микроэлектроники сделали возможным использование регулируемых асинхронных электроприводов как альтернативу электроприводам постоянного тока.

Однако есть области, где электроприводы постоянного тока незаменимы, поскольку их быстродействие в разы выше частотных асинхронных электроприводов, например, реверсивные электроприводы прокатных станов, в которых быстродействие является главенствующим фактором, определяющим их производительность. На электрическом транспорте продолжают широко использоваться двигателя постоянного тока последовательного возбуждения, которые обеспечивают работу с постоянной мощностью $[3,4] . \quad$ В электромобилях постоянного тока для регулирования скорости используются широтно-импульсные преобразователи. В электромобилях переменного тока для регулирования скорости используются автономные инверторы. При этом потери в широтноимпульсном преобразователе в разы меньше, чем в автономном инверторе частоты. Поэтому продолжают появляться публикации на тему электроприводов постоянного тока. В $[5,6]$ рассмотрены режимы рекуперативного торможения электромобиля постоянного тока с применение суперконденсаторов. При этом обеспечивается высокий тормозной момент, поскольку суперконденсаторы допускают высокую скорость зарядки. Увеличивается срок эксплуатации аккумуляторов, обеспечивается значительная экономия энергии аккумулятора и увеличивается пробег электромобиля до зарядки. В [7] рассмотрен тиристорный электропривод постоянного тока с оптимальным управлением переходными процессами с модальным наблюдателем.

Продолжаются публикации и по энергетике вентильных электроприводов постоянного тока, поскольку двигатели потребляют пульсирующий ток, хотя изготавливаются для питания напряжением без пульсаций. При этом гармоники тока якоря, нагревая обмотку, не создают крутящего момента на валу двигателя. Это означает, что при номинальной электрической мощности двигателя необходимо уменьшать механическую мощность на валу для обеспечения номинального температурного режима обмотки якоря. Следует отметить, что в 
настоящее время в каталогах можно найти специальные двигатели постоянного тока для подключения к полупроводниковым преобразователям, которые дороже двигателей общепромышленного назначения примерно на 30\%, поскольку имеют увеличенный диаметр проводов обмоток и увеличенную поверхность охлаждения. В таком случае следует проводить техникоэкономическое сравнение: либо специальный двигатель, либо двигатель общепромышленного назначения ближайшей большей мощности.

\begin{tabular}{ccr} 
Двигатели & \multicolumn{2}{r}{ постоянного } \\
подключаются & к & неуправляемым \\
выпрямителям, & к & тиристорным
\end{tabular}
преобразователям однофазным или трехфазным и к транзисторным широтноимпульсным преобразователям. Выходное напряжение полупроводниковых преобразователей может быть любой формы, поскольку представляется рядом Фурье, содержащим постоянную и гармонические составляющие. Поэтому далее для краткости используем термин «полигармоническое питание».

Публикации по энергетике полупроводниковых электроприводов в основном рассматривают влияние преобразователей на потери в двигателях. Например, в [8] предложена модель для расчета потерь в двигателях постоянного тока c полигармоническим питанием с учетом нелинейного характера кривой намагничивания. Аналогичная модель рассмотрена в [9] для тягового электропривода троллейбуса с двигателями смешанного возбуждения и транзисторным преобразователем постоянного тока. В [10] проведено моделирование потерь от высших гармоник в магнитопроводах электрических тяговых двигателей. В [11] исследовано влияние толщины листов электротехнической стали на потери от вихревых токов при полигармоническом питании. В [12] предложена тепловая карта нагрева тяговых электродвигателей при пульсирующем питании. В [13] рассмотрены вопросы оценки потерь мощности при пульсирующем питании в зависимости от частоты коммутации и ширины импульсов транзисторного преобразователя. В [14] рассмотрена усовершенствованная модель потерь мощности импульсного тягового двигателя электровоза. В $[15,16]$ рассмотрены зависимость коэффициента пульсаций напряжения от параметра управления преобразователем тягового двигателя с постоянными магнитами на редкоземельных металлах с высокой плотностью мощности.

В [17] рассмотрены вопросы провалов крутящего момента в двигателях постоянного тока с постоянными магнитами из-за неидеальности их характеристик.

Таким образом, в публикациях по энергетике не рассмотрено влияние пульсаций тока на механическую мощность двигателя, что не позволяет обоснованно производить выбор его мощности при питании от преобразователя.

Целью работы является разработка методики определения механической мощности двигателей постоянного тока общепромышленного назначения при их питании от полупроводниковых преобразователей, поскольку при этом гармонические составляющие тока якоря не создают крутящего момента на валу.

\section{І. МЕТОДЫ ИССЛЕДОВАНИЯ}

Механическая мощность на валу двигателя определяется коэффициентом полезного действия (КПД), который обычно определяют экспериментально в процессе запуска серии в производство путем расчета потерь мощности по формуле [18]

$$
\eta=1-\left(p_{\text {мx }}+p_{\text {мг }}+p_{\text {eл }}+p_{\partial}\right) / P_{1}
$$

где $p_{\text {мх }}, p_{\text {мг }}, p_{\text {ел }}, p_{\partial}, P_{1} \quad-\quad$ соответственно механические, магнитные, электрические, дополнительные потери и потребляемая электрическая мощность.

При полигармоническом питании в выражении (1) гармоники тока якоря увеличивает электрические потери $p_{\text {eл }}$ относительно питания без пульсаций, что эквивалентно уменьшению КПД двигателя и, следовательно, уменьшению механической мощности. Магнитные потери в стали якоря складываются из потерь на вихревые токи и из потерь на перемагничивание. Потери на вихревые токи определяются толщиной пластин магнитопровода, потери на перемагничивание зависят от ширины петли гистерезиса и от частоты перемагничивания, которая определяется частотой вращения якоря. Кроме того, в общем балансе 
магнитные потери примерно в пять раз меньше от электрических потерь, поэтому в данном случае не учитываются.

Для оценки влияния гармоник тока на эквивалентный КПД двигателя целесообразно использовать уравнение баланса мощностей электромеханического преобразования энергии. Для обобщенной активноиндуктивной нагрузки уравнение баланса мощностей при полигармонических токах приведено в [19], но для двигателей постоянного тока не может быть использовано, поскольку не содержит составляющей механической мощности.

В публикации [20] на основе дифференциальных уравнений баланса напряжений цепи якоря двигателя постоянного тока и баланса моментов, действующих на якорь, получено уравнение баланса мощностей. Но механическая мощность рассчитывается численным методом, то есть не приведено аналитического выражения для расчета.

Поэтому необходимо составить выражения для оценки влияния гармоник тока на механическую мощность. Для одного и того же значения механической мощности электрические потери при питании двигателя пульсирующим током будут больше чем при питании током без пульсаций, поскольку гармоники тока не создают крутящего момента (механической мощности). Определим увеличение мощности электрических потерь в обмотке якоря при питании пульсирующим током относительно мощности потерь при питании током без пульсаций (без гармоник)

$$
\begin{aligned}
& \Delta P_{n c} / \Delta P_{0}=\left(I_{0}^{2}+\sum I_{k}^{2}\right) / I_{0}^{2}= \\
& =\left\|\sum I_{k}^{2} / I_{0}^{2}=K_{n c}^{2}\right\|=1+K_{n c}^{2},
\end{aligned}
$$

где $I_{0}, \sum I_{k}$ - постоянная составляющая и сумма действующих значений гармоник тока якоря; $K_{n c}-$ коэффициент пульсаций тока якоря, как отношение суммы действующих значений гармоник тока к постоянной составляющей тока якоря, т.е., $K_{n c}=\sum I_{k} / I_{0}$.

Коэффициент пульсаций тока не зависит от мощности двигателя и в этом смысле является обобщенным параметром. Поскольку мощность электрических потерь при полигармоническом питании растет, следует пропорционально уменьшать механическую мощность, чтобы электрическая мощность двигателя не превысила номинального значения. Для количественной оценки необходимого уменьшения механической нагрузки на валу вводим понятие коэффициента $K_{м з}$ допустимой механической загрузки, как отношение механической мощности $P_{\text {мn }}$ при пульсирующем токе к механической мощности $P_{\mu 0}$ при токе без пульсаций. Для определения коэффициента $K_{\text {мз }}$ составим уравнение баланса мощностей

$$
P_{M 0}+I_{0}^{2} R_{Я}=P_{M n}+I_{0}^{2} R_{Я}\left(1+K_{n c}^{2}\right),
$$

из которого следует, что при увеличении коэффициента пульсаций тока необходимо уменьшать механическую мощность $P_{\text {мn }}$. Выполнив необходимые алгебраические преобразования, получим выражение для расчета коэффициента допустимой механической загрузки двигателя механической мощностью

$$
K_{\mathcal{M}}=\frac{P_{M n}}{P_{M 0}}=1-\frac{I_{0}^{2} R_{Я}}{P_{M 0}} K_{n c}^{2} .
$$

Таким образом, методика определения механической мощности двигателя общепромышленного назначения при его питании от полупроводникового преобразователя заключается в следующем: 1) на компьютерной модели определить коэффициент пульсаций тока якоря; 2) определить допустимый коэффициент загрузки по формуле (4); 3) определить величину допустимой механической мощности.

Таким образом, предложенная методика позволяет рассчитать снижение механической мощности на валу двигателя постоянного тока общепромышленного назначения, подключаемого к полупроводниковому преобразователю, и далее сравнить с расчетной мощностью рабочего механизма.

\section{II. РЕЗУЛЬТАТЫ И ОБСУЖДЕНИЕ}

Выражение (4), как результат исследования может быть использовано при 
выборе параметров фильтра на выходе полупроводникового преобразователя в развитие метода, предложенного в [21], а также для выпрямителей с ШИМрегулированием [22,23] для уменьшения коэффициента пульсаций напряжения.

Коэффициент пульсаций тока якоря зависит от: 1) силовой схемы полупроводникового преобразователя; 2) параметра управления преобразователем; 3) электромагнитной постоянной времени цепи якоря; 4) постоянной составляющей тока, определяемой моментом нагрузки на валу.

Аналитическую

зависимость коэффициента пульсаций тока от указанных параметров можно определить только экспериментально с последующей аппроксимацией полиномом, например, методом планирования эксперимента. Эксперимент может быть либо натурным на лабораторном стенде, либо компьютерным на модели. Поскольку двигатели постоянного тока относятся к классу детерминированных систем, в которых функциональные зависимости параметров точно описываются дифференциальными уравнениями, то целесообразно проводить компьютерный эксперимент.
В качестве примера практического использования предложенной методики определим коэффициент допустимой механической загрузки двигателя механизма подачи токарного станка при питании от однофазного реверсивного тиристорного выпрямителя серии ЭПУ2-2. Двигатель постоянного тока серии 4ПФ112S с номинальными данными: механическая мощность $\mathrm{P}=2$ кBт; напряжение обмотки якоря $\quad \mathrm{U}=220 \mathrm{~B} ; \quad$ частота вращения $\mathrm{n}=450$ об/мин; номинальный ток якоря $\mathrm{I}=14,5 \mathrm{~A} ;$ омическое сопротивление и индуктивность якоря $\mathrm{R}_{\mathrm{r}}=1,07 \mathrm{OM}$; $\mathrm{L}_{\mathrm{s}}=0,037$ Гн.

Для расчета коэффициента пульсаций тока методом компьютерного эксперимента в программном пакете Simulink [24] составлена модель тиристорного электропривода (рис. 1). Модель двигателя постоянного тока использована из библиотеки пакета Simulink с окном для задания параметров двигателя. Угол управления тиристоров задается блоками Pulse Generator. Действующее значение тока якоря определяется блоками signal rms, а постоянная составляющая блоком Magnitude signal.

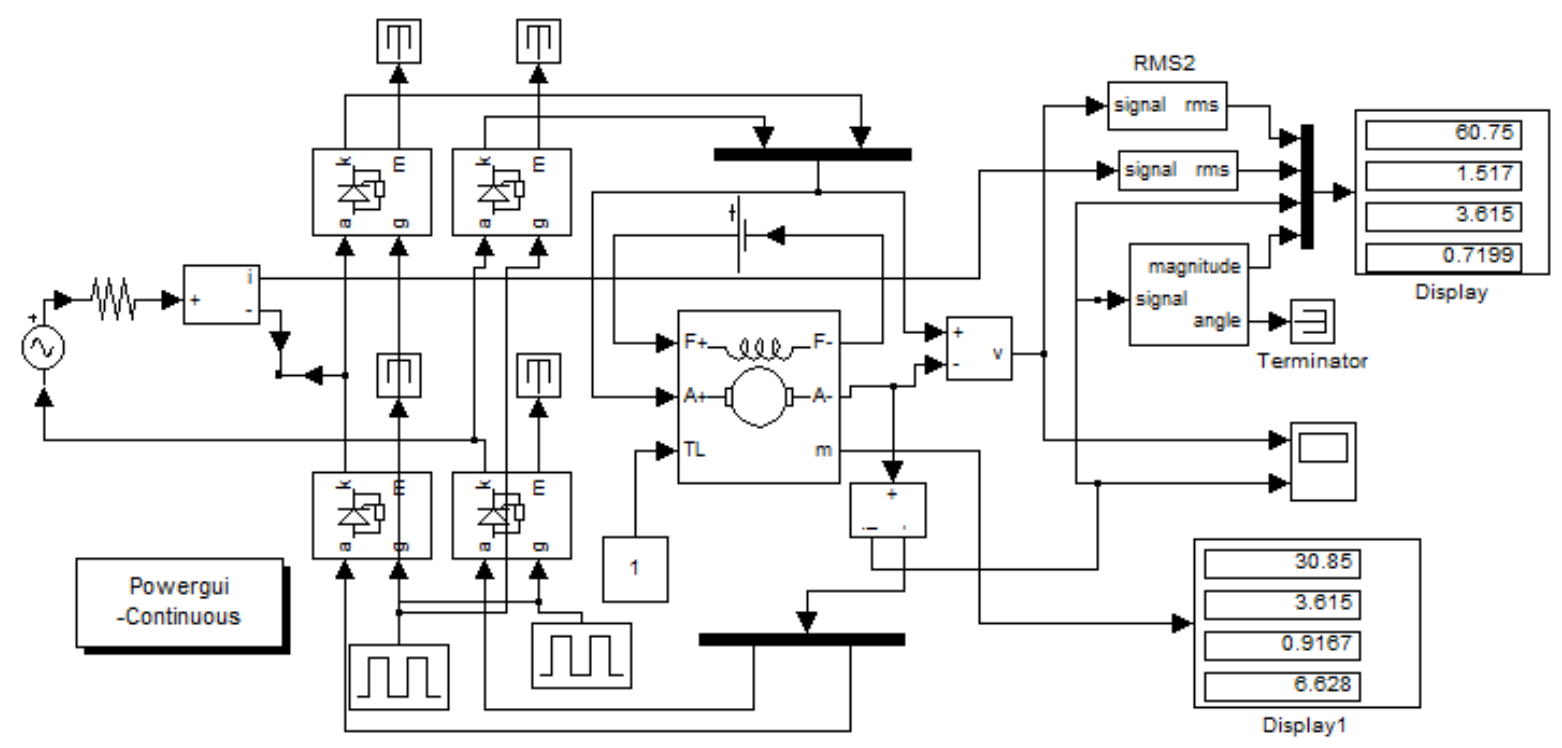

Рис. 1. Компьютерная модель электропривода с моделью двигателя из библиотеки Simulink. Fig. 1. Computer model electric drive with model DC motor with library.

Использование модели двигателя из библиотеки пакета Simulink не рационально, поскольку при этом рассчитываются переходные процессы, которые в данном случае не имеют никакого смысла и требуют значительное количество машинного времени. Поэтому целесообразно использовать модель двигателя постоянного тока в виде схемы замещения цепи якоря, представленной активным сопротивлением, 
индуктивностью и электродвижущей силой (ЭДС) обмотки якоря (рис. 2). Модель сразу выходит на установившийся режим и требует значительно меньшего времени для вычислений, что имеет значение при большом количестве экспериментов.

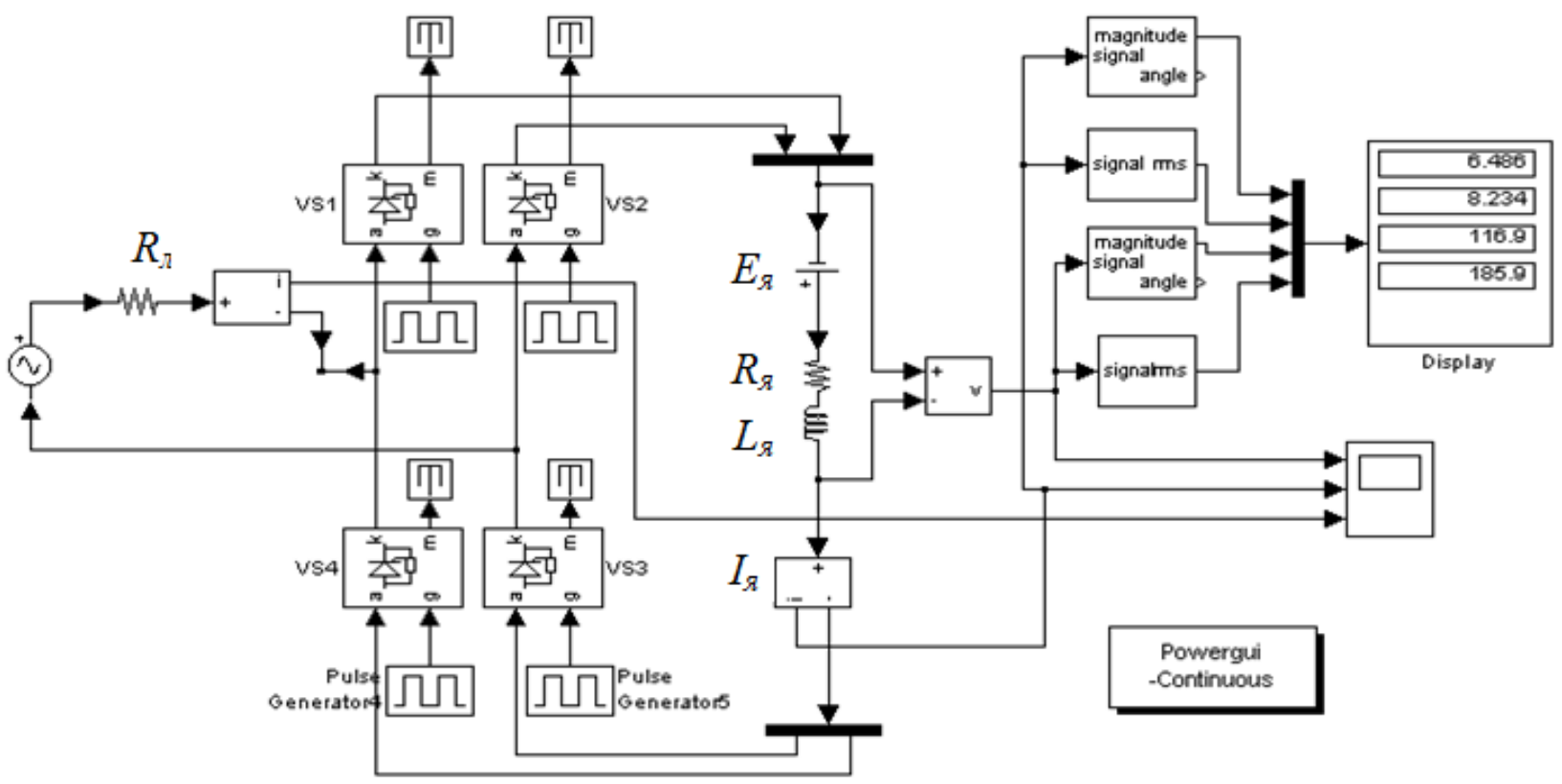

Рис. 2. Компьютерная модель с моделью двигателя в виде схемы замещения.

Fig. 2. Computer model electric drive with model DC motor in the form of a replacement circuit.

Для иллюстрации достоверности модели со схемой замещения были проведены эксперименты на лабораторном стенде. Осциллограммы напряжения и тока якоря, полученные на модели и на лабораторном стенде показаны на рис. 3.
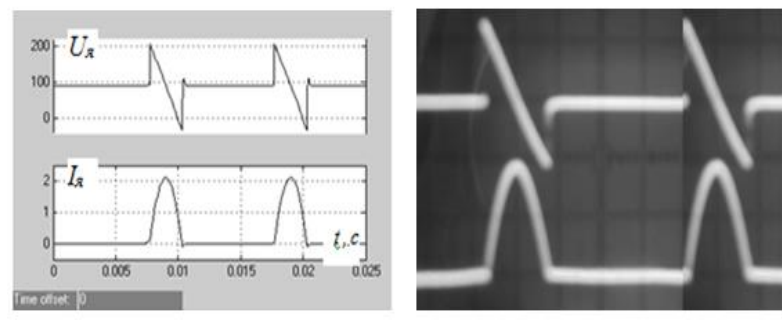

Рис. 3. Осциллограммы напряжения и тока якоря, полученные на модели и на лабораторном стенде.

Fig. 3. Oscillograms of armature voltage and current obtained on the model and on the laboratory stand.

Следует отметить, что такие же осциллограммы были получены и для модели на рис. 1 для установившегося режима.

В процессе компьютерного эксперимента на модели на рис. 2 необходимо получить зависимость коэффициента пульсаций тока от каждого из параметров при стабилизации двух других.

Стабилизация постоянной составляющей тока якоря (момент нагрузки) и ее изменение при компьютерных экспериментах реализуется путем изменения ЭДС якоря методом итераций вручную согласно формулы $I_{0}=\left(U_{0}-E_{a}\right) / R_{a}$. Блок $\mathrm{E}_{я}$ ЭДС якоря на схеме включен согласно с напряжением выпрямителя, но внутри блока задается со знаком «минус» из-за особенности включения блока в пакете Simulink. Активное сопротивление цепи якоря и его индуктивность задаются блоками $R_{\Omega}, L_{\Omega}$. Угол управления тиристоров задается задержкой импульсов Pulse Generator, электромагнитная постоянная времени задается изменением индуктивности цепи якоря, постоянная составляющая тока (момент нагрузки) задается изменением величины ЭДС якоря методом итераций вручную. Результаты компьютерных экспериментов в виде графиков показаны на рис. 4. 

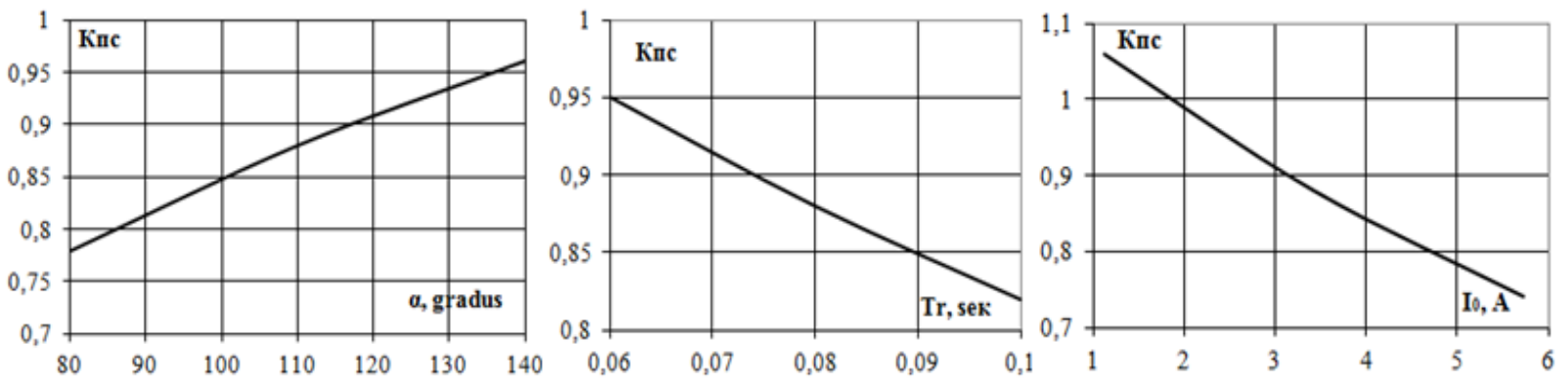

Рис. 4. Результаты компьютерных экспериментов в виде графиков.

Fig. 4. Results of computer experiments in the form of graphs.

Из графиков следует, что зависимости коэффициента пульсаций тока носят монотонный характер и поэтому могут быть аппроксимированы методом планирования эксперимента [25].

Функцией отклика (цели) является коэффициент пульсаций тока. Факторами являются параметры, которые изменяются в процессе эксперимента: угол управления тиристоров, электромагнитная постоянная времени цепи якоря, относительный ток нагрузки двигателя. Область эксперимента определяется максимальной $X_{\text {MAX }}$ и минимальной $X_{\text {мIN }}$ величинами факторов. Их кодирование состоит в том, что начало координат (точка $X_{0}$ ) факторного пространства переносится в центр эксперимента, т.е., $X_{0}=0,5\left(X_{\text {макс }}+X_{\text {мин }}\right)$. Интервал вариации факторов разбивается симметрично относительно $X_{0}$. Далее, согласно процедуре метода планирования эксперимента, получена аналитическая зависимость коэффициента пульсаций тока в виде полинома

$$
\begin{aligned}
& K_{n c}=0,89+0,19 \alpha-0,028 T_{g}-0,048 I_{o}^{*}+ \\
& +0,025 \alpha T_{\Omega}+0,015 \alpha I_{o}^{*}-0,05 T_{\Omega} I_{o}^{*}-0,003 \alpha T_{\Omega} I_{o}^{*}
\end{aligned}
$$

где $\alpha, T_{я}, I_{0}^{*}-$ соответственно угол управления тиристоров в радианах, электромагнитная постоянная времени якоря, постоянная составляющая тока якоря относительно номинального.

Относительная погрешность расчетных значений коэффициента пульсаций тока по формуле (5) зависит от комбинации факторов и в среднем равна «минус» $2 \%$. Далее, для электромагнитной постоянной времени. $T_{я}=0,06$ сек и относительного тока нагрузки
$I_{0}^{*}=0,74$ по выражению (5) рассчитывается зависимость коэффициента пульсаций тока от угла управления тиристоров и по выражению (4) рассчитана зависимость коэффициента допустимой механической загрузки двигателя от угла управления тиристоров, показанная на рис. 5.

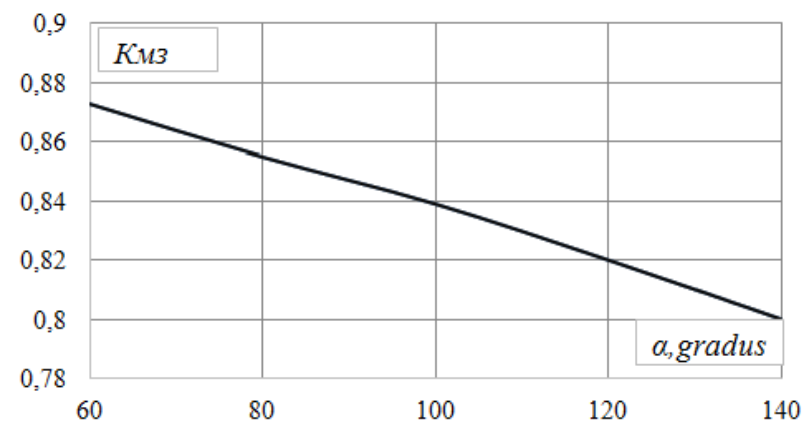

Рис. 5. Зависимость коэффициента допустимой механической загрузки двигателя от угла управления тиристоров.

Fig. 5. Dependence of the allowable mechanical load factor of the motor on the control angle of the thyristors.

На графике угол управления тиристоров, изменяется от 60 до 140 электрических градусов, что по статистике соответствует режиму работы механизма подачи суппорта токарного станка.

Известно [26], если индуктивность цепи якоря приближается к бесконечности, то углам управления в диапазоне $0^{0}<\alpha<90^{\circ}$ режим $90^{\circ}<\alpha<180^{\circ}-$ режиму инвертора. В случаях же конечного значения индуктивности диапазон режима выпрямителя превышает $90^{\circ}$. Это подтверждается экспериментальной осциллограммой (рис. 3), где отрицательные участки напряжения якоря соответствуют инверторному режиму. 


\section{III ВЫводЫ}

Таким образом, при выборе мощности двигателя постоянного тока общепромышленного назначения, подключаемого к тиристорному или транзисторному преобразователю, необходимо рассчитать снижение механической мощности на валу и сравнить с расчетной мощностью рабочего механизма.

Для этого предложена методика, которая заключается в следующем: 1) на компьютерной модели определить коэффициент пульсаций тока якоря; 2) по предложенному выражению определить коэффициент допустимой механической загрузки двигателя; 3) определить допустимую величину механической мощности.

При выполнении работы получило дальнейшее развитие выражение для определения допустимой механической загрузки двигателей постоянного тока при полигармоническом питании, которое, в отличие от известных, содержит квадрат коэффициента пульсаций тока якоря.

Приведен пример практического использования методики для расчета допустимой механической мощности двигателя постоянного тока при питании от однофазного тиристорного выпрямителя.

Методика может быть использована для всех типов и схем полупроводниковых электроприводов постоянного тока. Например, в транзисторном преобразователе электромобиля можно определить оптимальную частоту коммутации для обеспечения максимального коэффициента полезного действия (КПД) электропривода. Имеется виду, что механическая мощность двигателя с ростом частоты растет, то есть, КПД двигателя растет. Однако при этом растут потери в самом транзисторном преобразователе, то есть, КПД падает.

\section{Литература (References)}

[1] Paresh K. Sen. Electric motor drives and control past, present, and future. IEEE Transactions on Industrial Electronics, 1990, vol. 37, no. 6, pp. 562 - 575. doi: $10.1109 / 41.103462$.

[2] Vinod Kumar, Ranjan Kumar Behera, Dheeraj Joshi, Ramesh Bansal. Power Electronics, Drives and advanced Application. CRC Press, London. 2014. $509 \mathrm{p}$.
[3] Bimal K. Bose. Power Electronics and Motor Drives Advances and Trends. Tennessee Academic Press is an imprint of Elsevier, Burlington, USA 2006. 901 p.

[4] Federico Barrero, Jorge Rodas Control of Power Electronics Converters and Electric Motor Drives. Energies, 2021, no. 14, pp. 48-50.

[5] Klepikov V.B., Gonchar A.C. Regenerative modes of the electric drive of an electric vehicle with supercapacitors. Bulletin of the NTU "KhPI". Series: Problems of automated electrodrive. Theory and practice. - 2012. No. 3/2012 (19), pp. 216-218.

[6] Klepikov V.B., Semikov A.V. Energy efficiency of electric vehicle regenerative mode // Technical Electrodynamics. - 2017. - №6. - p. 36-42.

[7] Mikho Mikhov, Tsolo Georgiev. Control of DC Electric Drives Considering the Object Time Delay. International Journal of Science and Advanced Technology, 2013, vol. 3, no 8, pp 3238.

[8] Goolak S., Riabov Ie., Tkachenko V., Sapronova S., Rubanik I. Model of pulsating current traction motor taking into consideration magnetic losses in steel. Electrical Engineering \& Electromechanics, 2018, no. 6, pp. 11-17. doi: 10.20998/2074-272X. 2021.6.02

[9] Kharchenko V., Kostenko I., Liubarskyi B., Shaida V., Kuravskyi M., Petrenko O. Simulating the traction electric drive operation of a trolleybus equipped with mixed excitation motors and a DC-DC converter. Eastern-European Journal of Enterprise Technologies, 2020, vol. 3, no. 9 (105), pp. 46-54. doi: 10.15587/17294061.2020.205288.

[10] Rens J., Vandenbossche L., Dorez O. Iron Loss Modelling of Electrical Traction Motors for Improved Prediction of Higher Harmonic Losses. World Electric Vehicle Journal, 2020, vol.11, no 1, p. 24. doi: 10.3390/wevj11010024.

[11] Yamazaki K., Noiaki F. Torque and Loss Calculation of Rotating Mashines Considering Laminated Core. IEEE Transactions on Magnetics, 2011, vol. 47, no. 5, pp. 994 - 997. doi: 10.1109 / TMAG.2010.2089501

[12]Edison Gundabattini, Arkadiusz Mystkowski, Adam Idzkowski, Raja Singh R. and Darius Gnanaraj Solomon Thermal Mapping of a High-Speed Electric Motor Used for Traction Applications and Analysis of Various Cooling Methods - A Review. Energies 2021, no14, 1472. doi: 10.3390/en14051472.

[13] Yeongsu Bak, Kyo-Beum Lee. Reducing Switching Losses in Matrix Convertor Drives: Disconinuous PWM Method. Journal Power Electron (THE KOREEN INSTITUTE POWE ELECTONICS), 2018, vol. 18, no. 5, pp. 13251335.

[14] Goolak S., Sapronova S., Tkachenko V., Riabov I., Batrak Y. Improvement of the model of power 
losses in the pulsed current traction motor in an electric locomotive. Eastern-European Journal of Enterprise Technologies, 2020, vol. 6, no. 5 (108), pp. 38-46. doi: $10.15587 / 1729-$ $\underline{4061.2020 .218542 .}$

[15] Lingxiao Xue, Guj-Jia Su, Burak Ozpineci. DCRipple-Enerrgy Adaptive-Minimization Modulation Scheme for a High Power Density Convertor. IEEE Appled Power Electronics Conference (APEC). District of Columbia (USA), 2021, pp. 186-191.

[16] Tcarafidy Raminosoa, Randy Willis, Kevin Bannion. A High-Speed High-Power-Density Non-Heavy Rare-Earth Permanent Magnet Traction Motor. 2020 IEEE Energy Conversion Congress and Exposition (ECCE). Detroit, Michigan (USA), 2020, pp. 61-67.

[17] Usama Mohhmad Arafa. Elimination of torque dips due to back EMF non-ideality DC motors with surface-mounted magnets. Advanced Control of Electrical Drives and Power Electronics Convertor, 2015, Vol.2, no.1, pp. 2034. doi:10.1504/IJIED.2015.068767.

[18] Voldek A.I. Elektricheskiye mashini [Electrical mashines]. S. Petersburg: Piter. 2007. 272 p.

[19] Gzarnecki L Comments on Active Power Flow and Energy Accounts In Electrical Systems With Nonsinusoidal Waveforms and Asymmetry. IEEE Transaction on Power Divelery. 1996. Vol 11, No 3, P. 1244-1250.

[20] Bialobrzhesky O.V., Qawaqzeh M.Z., Zagimyak M.V., Rodkin D.Y., Gladyr A. I.. The analysis of the components of the power of a direct current motor armature circuit at periodic change of voltage. 2019 IEEE 2nd Ukraine Conference on

\section{Сведения об авторах.}

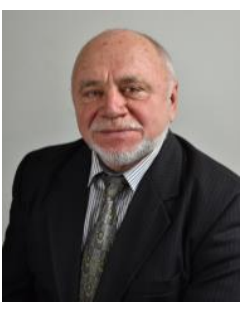

Виктор Николаевич Ковалев, кандидат технических наук, доцент, доцент кафедры автоматизированных

электромеханических систем Национального технического университета «Харьковский политехнический институт» E-mail: kovalov1952@gmail.com

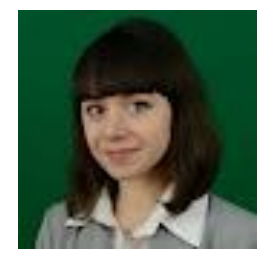

Юлия Викторовна Ковалева, кандидат технических наук, доцент, доцент кафедры систем электроснабжения и электропотребления городов Харьковского национального университета городского хозяйства имени А.Н. Бекетова E-mail: kovalova.jv@gmail.com
Electrical and Computer Engineering (UKRCON), Lviv, Ukraine, 2019, pp. 576-581. doi: 10.1109/UKRCON.2019.8879780.

[21] Mingfei Wu, Dylan Dan-Chuan Lu. A Novel Stabilization Method of LC Input Filter With Constant Power Louds Without Performance Compromise in DC Motor. IEEE Transactions on Industrial Electronics, 2015 vol. 62 , no. 7 , pp. 4552-4562. doi: 10.1109 / TIE.2014.2367005.

[22] Zargary N.P., Jus G. Performance investigation of current-controlled voltage-regulated PWM rectifier in rotating and stationary frames. IEEE Transactions on Industrial Electronics, _1995, Vol. 42 , no 4, pp. 396 - 401. doi: $\underline{10.1109 /}$ $\underline{41.402479}$

[23] Jee-Woo Lim, Bong-Hwan Kwon. A powerfactor controller for single-phase PWM rectifiers. IEEE Transactions on Industrial Electronics, 1999 vol. 46 , no. 5 , pp. 1035-1037. doi: $10.1109 / 41.793353$

[24] German-Galkin S.G. Comp'yuternoye modelirovaniye poluprovodnikovikh system $v$ MATLAB 6.0 [Computer model of the semiconductor systems in MATLAB 6.0]. S. Petersburg: CORONA. 2007. 320 p.

[25] Vlasov K.P. Metody issledovaniy $i$ organizatcii eksperimentov [The method of investigation and organization experimtnts] Kharkov: Gumanitarnyi center. 2002. 256 p.

[26] Chizhenko I.M., Rudenko V.S., Senko V.I. Osnovi preobrazovatelnoy tekhniky [Fundamentals of Conversion Technique]. M., "Visch. shkola", 1974. - 430 p.

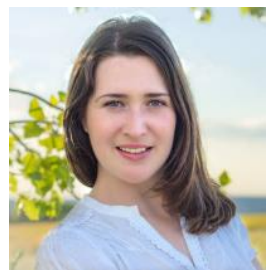

Ирина Евгеньевна Щербак, кандидат технических наук, старший преподаватель кафедры систем электроснабжения и электропотребления городов Харьковского национального университета городского хозяйства имени А.Н. Бекетова E-mail: ie.shcherbak@gmail.com 